\title{
Erratum zu: Enterprise Microblogging
}

DOl 10.1007/s1 1576-010-0253-z

\section{Die Autoren}

PD Dr. Kai Riemer ( $\varangle)$

Discipline of Business Information Systems

Faculty of Economics \& Business

University of Sydney

Building $\mathrm{H} 69$

Sydney

NSW 2006

Australia

kai.riemer@sydney.edu.au

\section{Dr. Alexander Richter}

Institut für Softwaretechnologie

Fakultät für Informatik

Universität der Bundeswehr München 85577 Neubiberg

Deutschland

a.richter@unibw.de

Dipl.-Wirt.-Inf. Martin Böhringer Professur für Wirtschaftsinformatik II, insb. Systementwicklung/

Anwendungssysteme

Fakultät für Wirtschaftswissenschaften Technische Universität Chemnitz 09126 Chemnitz

Deutschland

martin.boehringer@wirtschaft.

tu-chemnitz.de

Online publiziert: 2010-12-09

Die Online-Version des Originalartikels können Sie unter doi: 10.1007/s11576-010-0246-y finden.

(c) Gabler Verlag 2010
WIRTSCHAFTSINFORMATIK (2010) 52(6): 387-390

Der Nachname des Autors Böhringer wurde im Beitrag leider nicht korrekt wiedergegeben. Richtig ist: Dipl.-Wirt.-Inf. Martin Böhringer (nicht Bohringer). 